\title{
Accidente cerebrovascular: enseñanza, prevención y detección por los alumnos de Enseñanza Primaria Obligatoria Stroke: teaching, prevention and detection by compulsory primary school students

\author{
Rafael Ángel Castro Jiménez
} \\ Universidad de Córdoba (España)
}

\begin{abstract}
Resumen. Introducción: el ictus es un problema de salud de primer orden. Uno de los factores de demora en la atención es el desconocimiento de la enfermedad por la población. OBJETIVO: Determinar la eficacia de la enseñanza del accidente cerebrovascular en niños que están cursando educación primaria obligatoria (EPO) del colegio La Trinidad en Córdoba. Metodología: estudio observacional descriptivo. Mediante un cuestionario anónimo se indaga sobre los conocimientos que presentaban antes y después de una sesión formativa de 30 minutos de duración. Resultados: Doscientos ochenta y siete participantes, $146(50,9 \%)$ niños y $141(49,1 \%)$ niñas, divididos en 12 clases diferentes. Tras la intervención contamos con un número mayor de aciertos que de no aciertos en el cuestionario, y como $\mathrm{P}<0,01$ en la mayoría de las preguntas del rango 1 con respecto al rango 2 y 3, por lo que la mejor edad para la retención de conocimientos es el rango 2, de 9-10 años. Conclusión: este trabajo, a pesar de sus limitaciones, ha mostrado que la intervención educativa sobre el accidente cerebrovascular mejora el nivel de conocimientos de los alumnos.
\end{abstract}

Palabras claves: Accidente cerebrovascular, Educación, Ictus, Niño, Conocimiento.

\begin{abstract}
Introduction: stroke is a major health problem. One of the factors of delay in care is the population's ignorance of the disease. Objective: To determine the effectiveness of the teaching of cerebrovascular accident in children who are attending compulsory primary education (EPO) at La Trinidad school in Córdoba. MethodologY: descriptive observational study. Through an anonymous questionnaire, the knowledge they presented before and after a 30-minute training session is investigated. Results: Two hundred and eighty-seven participants, 146 (50.9\%) boys and 141 (49.1\%) girls, divided into 12 different classes. After the intervention, we had a higher number of correct answers than non-correct answers in the questionnaire, and as $\mathrm{P}$ $<0.01$ in most of the questions in range 1 with respect to range 2 and 3, so the best age for knowledge retention is rank 2, 9 10 years. Conclusion: this work, despite its limitations, has shown that educational intervention on stroke improves students' level of knowledge.
\end{abstract}

Key words: Stroke, Education, Stroke, Child, Knowledge.

\section{Introducción}

Los accidentes cerebrovasculares son la tercera causa de muerte mundial, y la primera de discapacidad física en adultos. En España, suponen unos 27.122 episodios anuales, siendo la segunda causa de muerte en hombres y la primera en mujeres. La presentación más frecuente es en la franja de mayores de 65 años (INE, 2016).

Generan un trastorno circulatorio-cerebral de aparición brusca, alterando transitoriamente el funcionamiento del encéfalo (López Espuela et al.,2011). La OMS, lo define como: «síndrome clínico de origen vascular, caracterizado por la aparición súbita de signos de afección neurológica focal o global, que perdura 24 horas o más, o incluso pueden llevar a la muerte».

Fecha recepción: 13-03-21. Fecha de aceptación: 03-01-22

Rafael Ángel Castro Jiménez

rafaelangelcastro@gmail.com
Las causas que originan el ictus pueden deberse a: rotura de un vaso, provocando una hemorragia o por disminución del flujo sanguíneo que recibe parte del cerebro. Se clasifican por tanto en:

- Ictus isquémico: alteración del encéfalo secundario a un trastorno del aporte circulatorio, por causa cualitativa: calidad de la sangre (anemia, trombocitemia, policitemia); o cuantitativa: cantidad de sangre aportada al encéfalo (trombosis, embolia, bajo gasto cardiaco). Se diferencia en dos tipos (Gonzáles Piña et al., 2016; Maeso Martínez, 2010):

o Ataque isquémico transitorio (AIT): menos de 24 horas de duración, son breves episodios de disfunción focal, como consecuencia del déficit de aporte sanguíneo en el territorio irrigado. Es reversible, al finalizar no existe déficit neurológico (Díez Tejedor, 2006).

o Infarto cerebral: duración superior a 24 horas, pudiendo producir un área de necrosis tisular, que 
provocan la muerte de las células cerebrales por falta de aporte de oxígeno y nutrientes. Existen distintos subtipos: aterotrombótico, cardioembólico, lacunar, de causa rara o indeterminada (Díez Tejedor, 2006; Díez Tejedor et al.,2001).

Ictus hemorrágico: se producen por la extravasación de sangre dentro del encéfalo, generado por rotura de un vaso sanguíneo, arterial o venoso (1020\%). Se clasifican como (Díez Tejedor et al.,2001):

o Hemorragia intracerebral: una arteria cerebral profunda se rompe esparciéndose sangre entre el tejido cerebral circundante, presionándolo y dañándolo. Existe un aumento de la presión intracraneal poniendo en peligro la vida.

o Hemorragia secundaria a malformación arteriovenosa (subaracnoidea): rotura de una aneurisma arterial, provocando una hemorragia entre la superficie cerebral y la parte interna del cráneo (Gonzáles Piña et al., 2016; Ustrell-Roig et al.; Ustrell-Roig et al, 2007).

Al ser una enfermedad prevalente, es importante conocer los factores de riesgo asociados fomentando la prevención primaria y secundaria, evitando las secuelas, que disminuyen la calidad de vida del paciente:

No modificables (edad, sexo, raza y antecedentes familiares)

Modificables, (hipertensión arterial, tabaquismo, sedentarismo, diabetes, obesidad, dislipemia...) (Maeso Martínez, 2010; Posadas-Ruiz, 2017; Matthias Oleñik, 2016; Jiménez Hernández, 2011; Guía de práctica clínica sobre la prevención primaria y secundaria del ictus, 2009). (Tabla 1)

Tabla 1.

Factores de riesgo (autoría propia).

\begin{tabular}{cl}
\hline \multicolumn{1}{c}{ Factores de riesgo no modificables } \\
\hline Edad & $\begin{array}{l}\text { Principal factor de riesgo no modificable, la incidencia de ictus se } \\
\text { duplica cada } 10 \text { años a partir de los } 55 \text { años. }\end{array}$ \\
\hline Sexo & Más frecuente en mujeres. \\
\hline $\begin{array}{c}\text { Antecedentes } \\
\text { familiares }\end{array}$ & Existe una relación incierta con el ictus. \\
\hline Asociado a un riesgo más elevado de ictus. \\
\hline Hipertensión arterial & $\begin{array}{l}\text { Factor más importante junto con la edad. Se potencia cuando se asocia a } \\
\text { otros factores de riesgo modificables. }\end{array}$ \\
\hline Tabaquismo y alcohol & $\begin{array}{l}\text { Contribuyen a aumentar el riesgo del ictus. El tabaquismo constituye un } \\
\text { importante factor de riesgo tanto para el ictus isquémico como para la } \\
\text { hemorragia cerebral. }\end{array}$ \\
\hline Diabetes y dislipemia & Incrementan el riesgo de ictus. \\
\hline Sedentarismo & $\begin{array}{l}\text { La práctica de ejercicio se asocia a una disminución de riesgo a la hora de } \\
\text { padecer un ictus. }\end{array}$ \\
\hline Obesidad & Tanto la abdominal como la general se asocian a un aumento del riesgo. \\
\hline
\end{tabular}

Si el ictus no pudo prevenirse, será importante reconocer los síntomas iniciales, reduciendo de forma inmediata el daño cerebral, así sus consecuencias pueden ser menores. Estos síntomas son:

Cefalea repentina, fuerte, sin causa conocida.

Pérdida de fuerza repentina.

Pérdida súbita de visión, parcial o total, uni o bilateral, o diplopia.

Alteración repentina del habla.

- Problemas repentinos al caminar, desequilibrio, inestabilidad o sensación de vértigo intenso.

Trastorno de la sensibilidad, acorchamiento de inicio brusco, en cara, brazo o pierna de un hemicuerpo (Egido, 1997; Maeso Martínez, 2010;Rodriguez Álvarez et al., 2018,13$)$.

Constituye una emergencia médica, desde el inicio de los síntomas y el reconocimiento aplicando escalas neurológicas, nos permiten una actuación precoz. Las escalas más habituales son:

Escala Cincinnati: escala prehospitalaria basada en una simplificación de la escala del National Institute of Health (NIHSS), cuyo objetivo es identificar pacientes con sospecha de ictus. Se valora la presencia de: asimetría facial, pérdida de fuerza en los brazos y disartria (tabla 2). Con un parámetro, $72 \%$ de posibilidades de sufrir un ictus, con tres esta probabilidad se eleva al 82\% (Maeso Martínez, 2010; Aranda Aguilar et al, 2012).

Escala National institute of Health Stroke Score (NIHSS): se debe aplicar al inicio y durante la evolución. Se puntua de 0 a 42 .

- Gravedad del ictus: Leve $<4$, Moderado $<16$, Grave < 25, Muy grave e» 25.

- Necesidad de tratamiento revascularizador: NIHSS entre 4 y 25.

-Tiene valor pronóstico. (Aranda Aguilar et al, 2012; Escala NIHSS, 2017; Alcaide Pérez et al, 2011).

- Escala de Rankin Modificada: evalúa los cambios en la actividad y el estilo de vida después del ictus (Alcaide Pérez et al, 2011, Escala Rakin Modificada, 2017).

Como se ha podido constatar, es un problema de salud de primer orden, los pacientes que sobreviven, sufren importantes secuelas limitando sus actividades de la vida diaria y su morbimortalidad ocasiona una gran carga económica, además, sufrimiento a pacientes y familiares.

La identificación precoz y la reacción adecuada ante los síntomas iniciales, son claves ante un ictus, para minimizar las secuelas. Sería importante formar a las personas, para saber identificar la sospecha (Estrategia en ictus del Sistema Nacional de Salud, 2009), con formación para la salud desde edades precoces.

La Escala Cincinnati, es ideal, ya que cualquier persona debería ser capaz de aplicarla, y comprobar en un solo minuto si está padeciendo o no un ACV.

Tras aplicarla y ante la sospecha de ictus, se debe de actuar con el máximo de celeridad llamando a los ser- 
vicios de emergencias (061/112), explicando: «nos encontramos ante una persona que presenta un ictus, he aplicado la escala de Cincinnati y tiene alterados los parámetros», indicamos dónde nos encontramos, quedándose al lado de esa persona, siguiendo las ordenes que le den.

Sería importante capacitar a las personas para saber actuar ante esta situación de emergencia tiempo dependiente, siendo clave para el tratamiento y recuperación posterior. Un factor de demora en la atención, es el desconocimiento de la enfermedad, siendo idóneo formar a la población desde edades tempranas.

Por ello en este estudio se ha querido determinar como objetivo principal: describir la eficacia de una intervención educativa sobre el ictus en alumnos de educación primaria obligatoria en un colegio de la provincia de Córdoba. Como objetivos secundarios, se establecieron:

- Analizar el nivel de conocimientos previos.

- Comparando nivel de conocimientos tras realizar la intervención.

- Comparando niveles de conocimiento por grupos y si existen diferencias de aprendizaje por rango de edad y curso.

- Comprender la importancia de un ACV y su actuación rápida.

Tabla 2.

\begin{tabular}{|c|c|}
\hline \multicolumn{2}{|c|}{ Escala de Cincinati (autoría propia) } \\
\hline $\begin{array}{c}\text { ASIMETRÍA FACIAL } \\
\text { (Haga que el paciente sonría o muestre los } \\
\text { dientes) }\end{array}$ & $\begin{array}{l}\text { - Normal: ambos lados de la cara se } \\
\text { mueven de forma simétrica. } \\
\text {-Anormal: un lado de la cara no se mueve } \\
\text { tan bien como el otro. }\end{array}$ \\
\hline LENGUAJE & $\begin{array}{l}\text { - Normal: el paciente utiliza palabras } \\
\text { correctas, sin balbucear. } \\
\text {-Anormal: el paciente al hablar arrastra las } \\
\text { palabras, utiliza palabras incorrectas o no } \\
\text { puede hablar }\end{array}$ \\
\hline $\begin{array}{c}\text { FUERZA EN LOS BRAZOS } \\
\text { (Haga que el paciente cierre los ojos y mantenga } \\
\text { los brazos estirados durante } 10 \text { segundos) }\end{array}$ & $\begin{array}{l}\text { - Normal: ambos brazos se mueven igual. } \\
\text { - Anormal: un brazo no se mueve o cae } \\
\text { respecto al otro. }\end{array}$ \\
\hline
\end{tabular}

\section{Método}

Es un estudio observacional descriptivo, con intervención antes y después de sesión formativa. Participaron en el estudio 287 alumnos, excluyendo un total de 5 alumnos por no rellenar los tres cuestionarios.

Se disponía de autorización paterna para cada alumno, para participar en actividades en el programa docente a cursar dentro de asignatura Natural Science.

Muestra formada por alumnos matriculados EPO (Colegio Trinidad de Córdoba). Quedarían excluidos los alumnos no matriculados en EPO y los que no realizaron el total de las intervenciones. El objetivo sería instruir en la identificación de un ACV basado en la escala de Cincinnati. La selección se realizó mediante muestreo aleatorio, no probabilístico. La intervención tuvo lugar en las clases de primaria.

Se definieron las siguientes variables:

a) Sociodemográficas: se estudio la relación con el resultado final, que es la adquisición de conocimientos en el ACV:

- Edad: variable cuantitativa.

- Sexo: variable categoría binaria: niño/niña

Curso: $1^{\circ}$ a $6^{\circ}$ de primaria

b) Temáticas: resultado final, adquisición de conocimientos valorados cuantitativamente y puntuación.

El cuestionario utilizado es de elaboración propia, basado en otros sobre el abordaje poblacional de los primeros auxilios, con 8 preguntas y tres opciones de respuesta. La redacción de las preguntas estuvo adaptado a la edad de los participantes, evaluando el conocimiento sobre el ACV de manera anónima.

Previamente se realizó una intervención de $30 \mathrm{mi}-$ nutos, para conocer el grado de aprendizaje y recuerdo de los conceptos. Tras ello se realizó una intervención a los 15 y 30. Se les presentaba de forma básica y sencilla, el significado del ACV, tipos y factores de riesgo, concienciándolos de que algunos pueden ser modificables y la importancia de una vida saludable. Con la escala aprendían como detectar los síntomas de un ACV, y haciendo hincapié en el número telefónico de emergencias al que deberían de llamar, ya que es una enfermedad tiempo-dependiente de acceso a la atención sanitaria. Tras ello, se les mostró un video educativo adaptado a su edad, el cual, a través de una canción, resume los tres puntos básicos de la escala Cincinnati.

Se generó con los resultados un base de datos utilizando el procesador estadístico SPSS v25, realizando un análisis descriptivo de las variables junto con los resultados

a) cualitativas: las frecuencias relativas y absolutas

b) cuantitativas: la media, los valores mínimos y máximos junto con la desviación estándar.

\section{Resultados}

Los 287 alumnos participantes en el estudio, se distribuían por sexo de la siguiente forma: 146 (50,9\%) niños y $141(49,1 \%)$ niñas, pertenecientes al Colegio Trinidad de la ciudad de Córdoba (España).

En primer lugar, se abordó con los alumnos que cursaban de primero a sexto de la educación primaria obligatoria, el conocimiento sobre la enfermedad cerebrovascular o ictus, siendo bajo (13,9\%) (tabla 3), con las diferentes intervenciones mejoraba el conoci- 
Tabla 3

Pregunta 1 (distribución en porcentajes)

\begin{tabular}{lccc}
\hline & \multicolumn{3}{c}{ Encuesta } \\
\cline { 2 - 4 } & Primera & Segunda & Tercera \\
\hline Habían recibido información & $13,9 \%$ & $90,2 \%$ & $96,5 \%$ \\
No habían recibido información & $63,1 \%$ & $4,2 \%$ & $1 \%$ \\
Sin formación, pero si habían oído hablar & $23 \%$ & $5,6 \%$ & $2,4 \%$ \\
\hline
\end{tabular}

miento sobre esta enfermedad hasta alcanzar el 96,5\% siendo muy significativa la intervención en este aspecto. Además, mejoró la capacidad para definir el ictus pasando de un $66,2 \%$ inicial a un $81,2 \%$, aunque en este concepto disminuía respecto a la segunda intervención (tabla 4), pero si fue significativo el hecho de que hasta un $40,1 \%$ aprendieron a diferenciar entre los dos tipos de ictus según su etiología.

Llamaba la atención que un alto porcentaje identificaban correctamente el número de emergencias sanitarias $80,8 \%$, mejorando de manera significativa y alcanzado el 95,5\% tras la tercera intervención, algo relevante ya que en todo proceso tiempo dependiente es crucial conocer donde alertar los recursos de cara al abordaje de la patología.(tabla 4)

Uno de los objetivos fue que los alumnos fueran capaces de detectar de forma rápida y sencilla la posibilidad de aparición de un ictus mediante la aplicación de la Escala de Cincinati, siendo capaces de hacerlo al inicio el 35,9\% y evolucionando tras la tercera intervención el 72,5\% de los alumnos que cursaba EPO. Lo que hizo que estos alumnos reconocieran la parálisis de cara, la pérdida de fuerza en un miembro superior o alteraciones en el habla en el 83,3\% de ellos, siendo significativo ya que al inicio era reconocido por el 51,6\% de los alumnos(tabla 4).

Tabla 4

\begin{tabular}{|c|c|c|c|}
\hline \multicolumn{4}{|c|}{ Encue } \\
\hline & Primera & Segunda & Tercera \\
\hline \multicolumn{4}{|c|}{$\begin{array}{l}\text { 2.¿¿Sabes cuáles son los números de emergencias a los que debes llamar si alguien se pone } \\
\text { enfermo? Respuesta correcta: A. } 1120061\end{array}$} \\
\hline Aciertos & $80,8 \%$ & $98,6 \%$ & $95,5 \%$ \\
\hline Fallos & $18,8 \%$ & $1,4 \%$ & $4,5 \%$ \\
\hline No Contesta & $0,3 \%$ & $0 \%$ & $0 \%$ \\
\hline \multicolumn{4}{|c|}{$\begin{array}{l}\text { 3.¿Qué es un ICTUS o accidente cerebral? Respuesta correcta: A. Es una alteración en el flujo } \\
\text { de sangre que llega al cerebro }\end{array}$} \\
\hline Aciertos & $66,2 \%$ & $85 \%$ & $85 \%$ \\
\hline Fallos & $33,5 \%$ & $15 \%$ & $15 \%$ \\
\hline No contesta & $0,3 \%$ & $0 \%$ & $0 \%$ \\
\hline \multicolumn{4}{|c|}{$\begin{array}{l}\text { 4. En qué escala nos basamos para identificar el ICTUS? Respuesta correcta: B. Escala de } \\
\text { Cincinnati. }\end{array}$} \\
\hline Aciertos & $66,2 \%$ & $85 \%$ & $85 \%$ \\
\hline Fallos & $33,5 \%$ & $15 \%$ & $15 \%$ \\
\hline No contesta & $0,3 \%$ & $0 \%$ & $0 \%$ \\
\hline \multicolumn{4}{|c|}{$\begin{array}{l}\text { 5.¿ Cuáles son los síntomas del ictus? Respuesta correcta: A. Parálisis de cara, pérdida de fuerza } \\
\text { y problemas en el habla }\end{array}$} \\
\hline Aciertos & $51,6 \%$ & $89,5 \%$ & $83,3 \%$ \\
\hline Fallos & $48,1 \%$ & $10,5 \%$ & $16,7 \%$ \\
\hline No contesta & $0,3 \%$ & $0 \%$ & $0 \%$ \\
\hline \multicolumn{4}{|c|}{$\begin{array}{l}\text { 6.A la hora de identificar el síntoma de debilidad en la cara, ¿en que nos tenemos que fijar? } \\
\text { Respuesta correcta: B. En la boca, le pedimos que sonría. }\end{array}$} \\
\hline Aciertos & $27,7 \%$ & $84,3 \%$ & $82,2 \%$ \\
\hline Fallos & $74,6 \%$ & $15,7 \%$ & $17,8 \%$ \\
\hline No contesta & $1,7 \%$ & $0 \%$ & $0 \%$ \\
\hline \multicolumn{4}{|c|}{$\begin{array}{l}\text { 7.Pueden influir los hábitos de vida en el ictus?. Respuesta correcta: A. Si, debemos de seguir } \\
\text { unos hábitos de vida saludable para así poder evitarlo. }\end{array}$} \\
\hline Aciertos & $72,8 \%$ & $83,3 \%$ & $78,7 \%$ \\
\hline Fallos & $26,9 \%$ & $16,7 \%$ & $21,2 \%$ \\
\hline No contesta & $0,3 \%$ & $0 \%$ & $0 \%$ \\
\hline
\end{tabular}

Otro objetivo claro, era que los alumnos identificaran los hábitos saludables, como prevención primaria para la aparición de un ictus. Es importante recalcar que era alto el porcentaje de inicio en el conocimiento de los mismo un 72,8\% (tabla 4) no llegando a ser significativa su mejora posterior en las diferentes intervenciones, puede deberse a la implicación del AMPA del Colegio en los hábitos de vida, complementando la formación del alumnado

Por otro lado, con el objetivo de ver cuál es la edad de los participantes más adecuada para la intervención y que retengan los conocimientos, reagrupamos la muestra edad en tres rangos: 1 (6-8 años), 2 (9-10 años) y 3 (11-13 años), correspondiente a los diferentes ciclos que existen en los cursos de EPO (tabla 5). Se realiza una comparación de las medias mediante la prueba «t student» para muestras independientes con un intervalo de confianza del 95\%, en la que se agruparon las variables y se comparó el grupo 1 con respecto el 2 y el 3 , y el grupo 2 con respecto al grupo 3.

Varias de las cuestiones que se abordaron fueron: fue el saber cuales eran los números de emergencias a los que debes de llamar si alguien se pone enfermo, conocer que es un ictus, cuáles son los síntomas de ictus donde se observó estadísticamente que $\mathrm{p}<0,01$ en el grupo 1 con respecto el grupo 2 y 3.

Respecto a conocer que es un ictus, los grupos comparados presentaron una $\mathrm{p}<0,01$ en el grupo 1 con respecto el grupo 2 y 3 . Sin embrago en el conocimiento de los tipos de ictus que podíamos identificar, la escala para detectar un ictus, en como identificar el síntoma de debilidad de la cara, no existieron diferencias signifi-

\begin{tabular}{|c|c|c|c|c|}
\hline & Encuesta & & & \\
\hline & Primera & Segunda & Tercera & \\
\hline \multicolumn{5}{|c|}{$\begin{array}{l}\text { 8.- ¿Sabes cuales son los números de emergencias a los que debes de llamar si alguien se pone } \\
\text { enfermo? }\end{array}$} \\
\hline Grupo $1\left(1^{\circ}-2 \mathrm{EPO}\right)$ & $70,90 \%$ & $98,50 \%$ & $95,5 \%$ & \multirow{3}{*}{$\begin{array}{l}\mathrm{p}<0,01 \text { en grupo } 1 \text { con } \\
\text { respecto grupo } 2 \text { y } 3\end{array}$} \\
\hline Grupo $2\left(3^{\circ}-4^{\circ} \mathrm{EPO}\right)$ & $87,60 \%$ & $98 \%$ & $4,5 \%$ & \\
\hline Grupo $3\left(5^{\circ}-6^{\circ} \mathrm{EPO}\right)$ & $97,90 \%$ & $100 \%$ & $0 \%$ & \\
\hline \multicolumn{5}{|c|}{ 9.-¿Qué es un ictus o un accidente cerebro vascular?". } \\
\hline Grupo $1\left(1^{\circ}-2 \mathrm{EPO}\right)$ & $58,60 \%$ & $80 \%$ & $72,20 \%$ & \multirow{3}{*}{$\begin{array}{l}\mathrm{p}<0,01 \text { en grupo } 1 \text { con } \\
\text { respecto grupo } 2 \text { y } 3\end{array}$} \\
\hline Grupo $2\left(3^{\circ}-4^{\circ} \mathrm{EPO}\right)$ & $80,40 \%$ & $89,90 \%$ & $86 \%$ & \\
\hline Grupo $3\left(5^{\circ}-6^{\circ} \mathrm{EPO}\right)$ & $61,20 \%$ & $88,70 \%$. & $93,40 \%$. & \\
\hline \multicolumn{5}{|c|}{ 10.- $\_$Cuántos tipos de ictus podemos identificar?. } \\
\hline Grupo $1\left(1^{\circ}-2 \mathrm{EPO}\right)$ & $25,2 \%$ & $32,6 \%$ & $29,3 \%$ & \multirow{3}{*}{$\begin{array}{l}\mathrm{p}>0,01 \text { para grupo } 1,2, \\
3\end{array}$} \\
\hline Grupo $2\left(3^{\circ}-4^{\circ} \mathrm{EPO}\right)$ & $19,1 \%$ & $51,5 \%$ & $50,5 \%$ & \\
\hline Grupo $3\left(5^{\circ}-6^{\circ} \mathrm{EPO}\right)$ & $17 \%$ & $37,7 \%$ & $49,5 \%$ & \\
\hline \multicolumn{5}{|c|}{ 11. ¿En qué escala nos basamos para identificar el ictus? } \\
\hline Grupo $1\left(1^{\circ}-2 \mathrm{EPO}\right)$ & $29,9 \%$ & $71,1 \%$ & $70,7 \%$ & \multirow{3}{*}{$\mathrm{p}>0,01$ para grupo 1,2 y 3} \\
\hline Grupo $2\left(3^{\circ}-4^{\circ} \mathrm{EPO}\right)$ & $43 \%$ & $84,8 \%$ & $80,6 \%$ & \\
\hline Grupo $3\left(5^{\circ}-6^{\circ} \mathrm{EPO}\right)$ & $50 \%$ & $73,6 \%$ & $63,9 \%$ & \\
\hline \multicolumn{5}{|c|}{ 12.-Cuáles son los síntomas del ictus? } \\
\hline Grupo $1\left(1^{\circ}-2 \mathrm{EPO}\right)$ & $41,4 \%$ & $81,5 \%$ & $71,4 \%$ & \multirow{3}{*}{$\begin{array}{l}\mathrm{p}<0,01 \text { en el grupo } \\
\text { frente al grupo } 2 \mathrm{y}^{3}\end{array}$} \\
\hline Grupo $2\left(3^{\circ}-4^{\circ} \mathrm{EPO}\right)$ & $62,9 \%$ & $96 \%$ & $92,5 \%$ & \\
\hline Grupo $3\left(5^{\circ}-6^{\circ} \mathrm{EPO}\right)$ & $59,2 \%$ & $98,1 \%$ & $95,1 \%$ & \\
\hline \multicolumn{5}{|c|}{ 13.-A la hora de identificar el síntoma de debilidad en la cara, ¿en qué nos tenemos que fijar?. } \\
\hline Grupo $1\left(1^{\circ}-2 \mathrm{EPO}\right)$ & $32,4 \%$ & $78,5 \%$ & $75,9 \%$ & \multirow{3}{*}{$\begin{array}{l}\mathrm{p}>0,01 \text { tanto para } \\
\text { grupo } 1,2 \mathrm{y} 3 .\end{array}$} \\
\hline Grupo $2\left(3^{\circ}-4^{\circ} \mathrm{EPO}\right)$ & $22,7 \%$ & $90,9 \%$ & $89,2 \%$ & \\
\hline Grupo $3\left(5^{\circ}-6^{\circ} \mathrm{EPO}\right)$ & $4,1 \%$ & $86,8 \%$ & $85,2 \%$ & \\
\hline \multicolumn{5}{|c|}{ 14.-¿Puede influir los hábitos de vida en el ictus?. } \\
\hline Grupo $1\left(1^{\circ}-2 \mathrm{EPO}\right)$ & $74,3 \%$ & $85,2 \%$ & $84,2 \%$ & $\mathrm{p}<0,01$ en el grupo 1 con \\
\hline Grupo $2\left(3^{\circ}-4^{\circ} \mathrm{EPO}\right)$ & $74,2 \%$ & $84,8 \%$ & $81,7 \%$ & respecto al $3 \mathrm{y}$ en el grupo \\
\hline Grupo $3\left(5^{\circ}-6^{\circ} \mathrm{EPO}\right)$ & $67,3 \%$ & $75,5 \%$ & $62,3 \%$ & 2 con respecto al 3. \\
\hline
\end{tabular}


cativa, con una $\mathrm{P}>0,01$ tanto para el grupo 1, 2 y 3.

Finalmente cabe reseñar que respecto a cómo pueden influir los hábitos de vida en el ictus, se presentaron diferencias significativas entre el grupo 1 con respecto al 3 y el grupo 2 con respecto al 3.

\section{Discusión}

La «Educación para la Salud», se sustenta en los derechos fundamentales a la educación y a la salud, reconocidos en el marco constitucional (artículo 43). Se considera en la actualidad, como un elemento importante en la formación y con un objetivo el promover la salud. La escuela es el lugar idóneo para favorecer un crecimiento armónico de la personalidad del alumno, beneficiando su proceso evolutivo y este por lo tanto beneficiando el efecto en la sociedad.

Es por ello, que intentamos testar los conocimientos previos sobre el ictus, como proceso tiempo dependiente relevante para la salud y determinar, si existe eficacia de la intervención educativa en un rango de edad.

En los resultados obtenidos, la mayoría de las cuestiones de la primera encuesta, obtenemos un número mayor de no aciertos, y como en la segunda y tercera encuesta, tras impartir la intervención, el número de aciertos se eleva, asumiendo que la intervención ha sido efectiva. Observamos como en la segunda encuesta, hay un porcentaje $>3-4$ puntos respecto a la 3 encuesta, indicando que los alumnos responden mejor al cuestionario en la segunda encuesta, ya que ha pasado menos tiempo, por lo que debemos de seguir una dinámica en la enseñanza e ir recordando la materia (reciclaje), para que afiancen esos conocimientos y no sean olvidados con el tiempo.

Al no existir estudios relevantes sobre el nivel de conocimiento que presentan los niños escolarizados sobre dicho tema, lo comparamos con el estudio «Conocimiento y actitud de la población general frente al ictus» de Jiménez et al.(2015) existiendo un déficit de conocimiento del término y de la enfermedad, así como de la actitud ante los síntomas leves. Es por ello, que concluye afirmando que sería necesaria intervenciones educativas para mejorar la formación.

Otro artículo a comparar es el «Estudio sobre el conocimiento de la población acerca del ictus y de los factores de riesgo vascular» de Pérez-Lázaro et al. (2009), estudia el conocimiento que tiene la población del ictus y de los factores de riesgo, y la actitud ante éste. Existe un escaso conocimiento en la población del ictus y los factores de riesgo vascular, concluyendo de igual que el anterior, reforzando la importancia de campañas informativas para mejorar la actitud ante la enfermedad.

Debido al desconocimiento existente, es necesario implementar en los colegios la formación sobre el ictus y los riesgos que conllevan. Un mínimo de conocimiento, al ser una enfermedad común y tiempo-dependiente, sería fundamental para poder salvar la vida. Además, es la mejor etapa para enseñarles nociones básicas de esta y otras enfermedades, ya que retienen más información.

No obstante, esta debería ser impartida por un profesional sanitario, ya que hoy en día los profesores son los que imparten clase sobre temas y no cuentan con la. Es por ello, la importancia de la figura de un sanitario en los colegios, ya no solo para integrar la salud en el sistema educativo, sino para controlar cualquier suceso agudo o crónico. En la actualidad, algunos países europeos cuentan ya con enfermería, mientras que en España pocos colegios son los que se benefician de esta figura.

Por otra parte, los porcentajes de aciertos de cada encuesta por rango de edad, demuestran como los alumnos presentan mejor capacidad de retención de conocimiento a mayor edad, siendo la mejor en aquellos alumnos con 9-10 años, que pertenecen al segundo ciclo de EPO.

Las limitaciones pueden ser:

- Realizar la intervención con una encuesta no validada, debido al escaso número de intervenciones en este ámbito, aunque se ha adaptado a todos los niveles educativos.

- El colegio no fue elegido al azar, sino por decisión de los formadores.

- El tiempo destinado para esta actividad parece ser escaso, en comparación con otros estudios. Podemos pensar que, a mayor periodo de tiempo utilizado en la intervención, mayores niveles de conocimiento se obtienen.

\section{Conclusiones}

La intervención educativa sobre el ictus mejora el nivel de conocimientos en los participantes de la actividad, siendo una intervención eficaz, donde la mejor edad para empezar a impartirla es el rango de 9-10 años, donde mejor se retienen los conocimientos.

Es una experiencia motivadora, interesante y aporta beneficios tanto a los alumnos como a los formadores. Por otro lado, aunque los programas conllevan trabajo y 
dificultades, los beneficios para la comunidad son muchos, ayudando a eliminar ese desconocimiento sobre el tema. Es necesario promover y desarrollar programas de este tipo en todas las escuelas.

\section{Referencias}

Alcaide Pérez, A. Alonso Muñoz, G. Aranda Aguilar, E. Bravo Rodríguez, F. Cañadillas Hidalgo, F. Castilla Camacho, S. Chirosa Ríos, MA. Delgado Acosta, F. Giráldez Rodríguez, A. Ochoa Sepúlveda, JJ. Oteros Fernández, R. Porras Pantojo, MF. Roldan Romero, E. 2011. Manual de Actuación en la Fase Aguda del Infarto Cerebral. Editorial: Imprenta San Pablo, SL. Córdoba. España.

Aranda Aguilar F, Borja Padilla J, Lobato Martinez R, Martín Pérez P. 2012. Procesos neurológicos. Málaga: Empresa Pública de Emergencias Sanitarias.

Díez Tejedor E, Del Bruto O, Alvarez Sabín J, Muñoz M, Abiusi G. 2001. Clasificación de las enfermedades cerebrovasculares. Sociedad Iberoamericana de Enfermedades Cerebrovasculares. Revista de Neurología. 33:455-64.

DíezTejedor E. 2006. Guía para el diagnóstico y tratamiento del ictus. 3rd ed. Madrid: Prous Science.

Egido J, Diez Tejedor E. 1997. Después del ictus. Barcelona: Sociedad Española de Neurología, Grupo de Estudio de las Enfermedades Cerebrovasculares.

Escala de Rankin Modificada.2017. Tiempo es Cerebro Sociedad Española de Medicina de Urgencias y Emergencias [Internet]. [citado 25 Abril 2018]:2. Disponible: http: / / tiempoescerebro.com/wpcontent/ uploads/2017/06/rankin-modificada.pdf

Escala NIHSS National Institute of Health Stroke Score. 2017. Tiempo es Cerebro Sociedad Española de Medicina de Urgencias y Emergencias [Internet]. [citado 25 Abril 2018]:4. Disponible en: http:// tiempoescerebro.com/wp-content/uploads/2017/ 06/nihss.pdf

Estrategia en ictus del Sistema Nacional de Salud. 2009. [Madrid]: Ministerio de Sanidad y Política Social.

Gonzáles Piña, R, Landínez Martínez, D. Epidemiología, etiología y clasificación de la enfermedad vascular cerebral. 2016. Archivos de Medicina (Col) [Internet]. 16(2):495-507.Recuperado de: http: / / www.redalyc.org / articulo. oa?id=273849945026

Guía de práctica clínica sobre la prevención primaria y secundaria del ictus. 2009. Barcelona: Agencia d'Avaluacio de Tecnologia i Recerca Mediques de Cataluna [i.e. Catalunya].
Instituto Nacional de Estadística (INE).2016. Notas de prensa [Internet]. Madrid: INE; [citado 12 abril 2018]. Disponible en: http://www.ine.es/prensa/ edcm_2016.pdf

Jiménez Gracia M, Amarilla Donoso J, Güesta Guerra E, Leno Diaz C, Portilla Cuenca J. 2015. Conocimiento y actitud de la población general frente al ictus. Revista Científica de la Sociedad Española de Enfermería Neurológica. ISSN 2013-5246. 15-21.

Jiménez Hernández M. 2011. Plan Andaluz de Atención al Ictus, 2011-2014. [Sevilla]: Consejería de Salud. Junta de Andalucía.

López Espuela F, Jiménez Gracia M, Luengo Morales E, Blanco Gazapo A, Márquez Caballero J, Bravo Fernández S et al. 2011. Estudio descriptivo de los pacientes asistidos en una unidad de ictus en la Comunidad de Extremadura. Enfermería Intensiva. 22(4):138-143.

Maeso Martínez S. 2010. Guía práctica clínica para el manejo de pacientes con ictus en atención primaria. Madrid: Ministerio de Ciencia e Innovación.

Matthias Oleñik C. 2016. Factores de riesgo cardiovascular en accidente cerebrovascular. Revista virtual de posgrado.1(1).

Pérez-Lázaro C. 2009. Estudio sobre el conocimiento de la población acerca del ictus y de los factores de riesgo vascular. Revista Neurología. 49 (3): 113-118.

Posadas-Ruiz L. 2017. Factores de riesgo asociados a accidente cerebrovascular: un problema de salud pública. Revista de la Facultad de Medicina Humana.17(3).

Rodríguez Álvarez S, Fernández Rodríguez EJ, Sánchez Gómez C. 2017. Estudio sobre la percepción de calidad de vida que presentan pacientes que han sufrido un ictus a través de la ECVI-38.TOG (A Coruña) [revista en Internet].[acceso: 25 Abril 2018]; 14(25): 148-58. Disponible en: http:// www.revistatog.com/num25/pdfs/original9.pdf

Ustrell-Roig X, Serena-Leal J. 2007. Ictus. Diagnóstico y tratamiento de las enfermedades cerebrovasculares. Revista Española de Cardiología. 60(7):753-769.

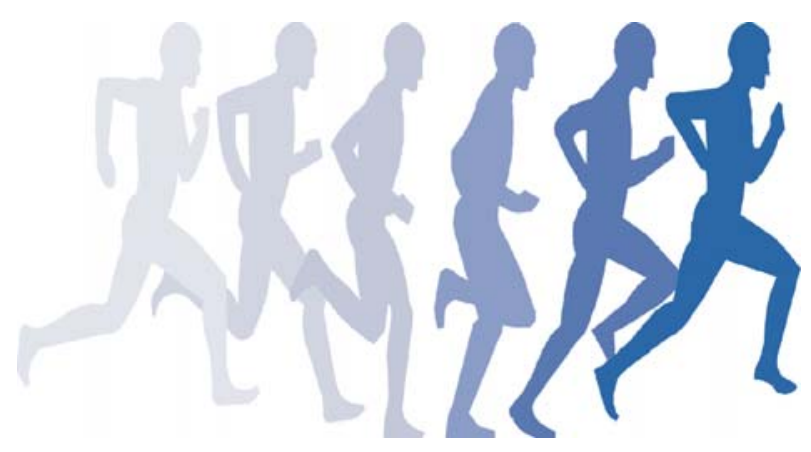

